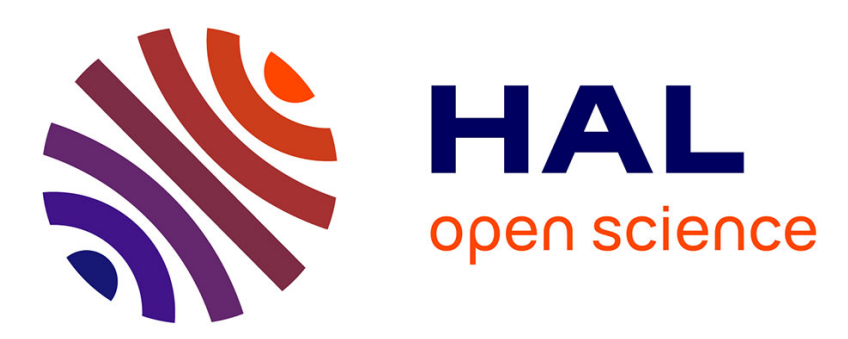

\title{
Electron Injection into the Gate Oxide of MOS Structures at Liquid Nitrogen Temperature: Measurement and Simulation
}

\author{
B. Fischer, L. Selmi, A. Ghetti, E. Sangiorgi
}

\section{- To cite this version:}

B. Fischer, L. Selmi, A. Ghetti, E. Sangiorgi. Electron Injection into the Gate Oxide of MOS Structures at Liquid Nitrogen Temperature: Measurement and Simulation. Journal de Physique IV Proceedings, 1996, 06 (C3), pp.C3-19-C3-24. 10.1051/jp4:1996303 . jpa-00254221

HAL Id: jpa-00254221

https://hal.science/jpa-00254221

Submitted on 1 Jan 1996

HAL is a multi-disciplinary open access archive for the deposit and dissemination of scientific research documents, whether they are published or not. The documents may come from teaching and research institutions in France or abroad, or from public or private research centers.
L'archive ouverte pluridisciplinaire HAL, est destinée au dépôt et à la diffusion de documents scientifiques de niveau recherche, publiés ou non, émanant des établissements d'enseignement et de recherche français ou étrangers, des laboratoires publics ou privés. 


\title{
Electron Injection into the Gate Oxide of MOS Structures at Liquid Nitrogen Temperature: Measurement and Simulation
}

\author{
B. Fischer, L. Selmi, A. Ghetti and E. Sangiorgi* \\ DEIS, University of Bologna, Viale Risorgimento 2, 40136 Bologna, Italy \\ * DIEGM, University of Udine, Via delle Scienze 208, 33100 Udine, Italy
}

\begin{abstract}
Measured electron injection probabilities $\left(P_{J N}\right)$ into the gate oxide of MOS structures [1] are presented for both liquid nitrogen and room temperature. For the first time $P_{J N}$ has been measured for total voltage drops $\left(V_{T O T}\right)$ in the substrate spanning from well below to well above the value corresponding to the $\mathrm{Si}_{-} \mathrm{SiO}_{2}$ barrier height $\left(V^{*}\right.$ TOT $\left.=\Phi_{B} / q=3.15 \mathrm{~V}\right)$. In contrast to MOSFET data [2], here two different injection regimes can clearly be distinguished. For $V_{T O T}<V^{*}$ TOT a regime occurs in which injection is no longer caused only by the electron energy gained in the electric field but also by the additional energy from phonon absorption. No lucky electron model holds true in this regime and therefore some insight will be given here by means of Monte Carlo simulations of the injection experiment. A simple analysis technique is developed, that explains the observed temperature dependence and injection regimes of $P_{J N}$.
\end{abstract}

\section{INTRODUCTION}

Power supplies of present day ULSI circuits are approaching $3.3 \mathrm{~V}$ or lower values, that is the critical threshold of $3.15 \mathrm{~V}$ corresponding to the $\mathrm{Si}_{-} \mathrm{SiO}_{2}$ barrier height. As a consequence, the energy of fieldheated electrons may not be sufficient for significant injection into the gate oxide. Additional energy-gain mechanisms are expected to cause the substantial hot-carrier effects still observed, thus raising new questions on the bias and temperature dependence of gate and substrate currents.

In this work we investigate the temperature dependence of low voltage gate currents in MOS structures as a way to identify and clarify the role of a particular energy-gain mechanism, namely phonon absorption from the lattice. In order to avoid the combined and device dependent effects inherent in the twodimensional MOSFET geometry and field distribution [1], as well as the competitive action of other energy-gain mechanisms such as electron-electron scattering [3], we focus on homogeneous injection experiments which are essentially one-dimensional and weakly affected by the electron-electron interaction.

\section{THE MEASUREMENT SETUP}

The structure used for injection is an n-MOS floating gate transistor (Fig. 1) with so-called re-entrant layout [1]. A homogeneous stream of photo-generated electrons from the bulk reaches the depletion region and is accelerated towards the semiconductor-oxide interface due to the high electric field inside. Some of them are eventually injected into the floating gate. The gate current is measured indirectly by the threshold voltage shift, leading to extremely sensitive measurements down to $10^{-18} \mathrm{~A}$. We never observed any degradation of the gate oxide, which means that almost no electrons are trapped in it. The injection probability $\left(P_{J N}\right)$ is equal to the ratio of the gate current to the total current that reaches the area under the 
gate [1]. Due to the re-entrant layout we can calculate the total current from the measured drain current considering a geometrical factor of the device structure [4].

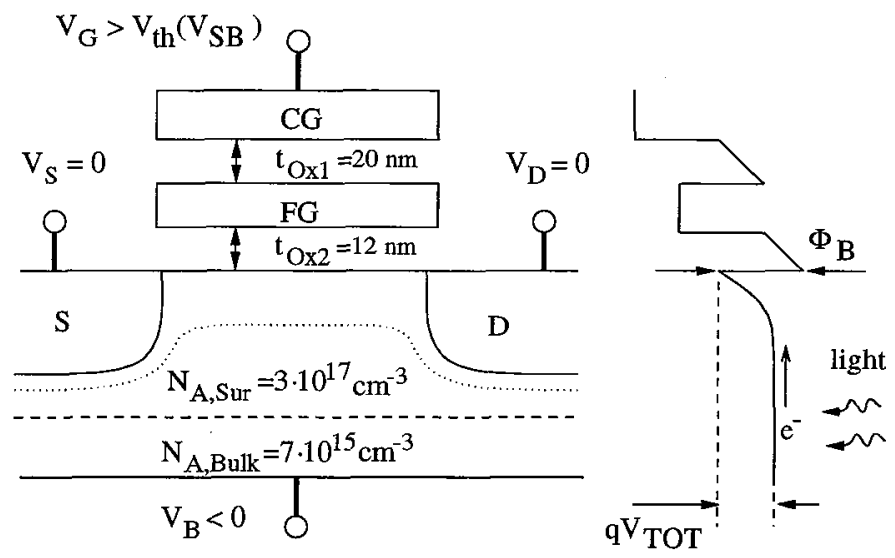

Figure 1: Schematic vertical cut through the n-MOS floating gate transistor used in the homogeneous injection experiment. $V_{t h}\left(V_{S B}\right)$ denotes the body bias dependent threshold voltage of the device.

\section{THE MEASUREMENTS}

Fig. 2 and Fig. 3 show $P_{J N}$ as a function of $V_{T O T}$ at $T=77 \mathrm{~K}$ and $T=300 \mathrm{~K}$ respectively. The boundary values of the oxide field $\left(F_{O x}\right)$ are given by the necessity that the device is still biased in inversion for the lowest value and tunneling of cold electrons is still small for the highest one. $V_{\text {TOT }}$ is calculated by PISCES-2ET [5] and approximately given by

$$
V_{T O T} \simeq-V_{B}+2 k T / q \ln \left(N_{A, S u r} / n_{i}\right)
$$

in which $k$ is the Boltzmann constant, $q$ the elementary charge, and $n_{i}$ the intrinsic carrier density. At both temperatures two different injection regimes are present: the high voltage regime with a shallow slope of the $P_{I N}$ curves, and the low voltage regime with a steep slope that depends on the oxide field (increasing slope with decreasing field). The transition region occurs around $V^{*}{ }_{T O T}=3.15 \mathrm{~V}$. This behavior is significantly different from that recently observed in MOSFETs [2], in which it is not possible to distinguish between different gate current regimes in spite of a drain voltage range comparable to the $V_{T O T}$ range explored here. Comparing directly the curves at different temperatures in Fig. 4, we observe that $P_{J N}$ at liquid nitrogen temperature is larger than at $300 \mathrm{~K}$ in the high voltage regime. This can be explained by the phonon emission scattering rate that dominates under the above conditions in Si. The number of scattering events in which electrons lose part of their energy by phonon emission is proportional to $\left(N_{\beta}+1\right)$ with

$$
N_{\beta}=1 /(\exp (\hbar \omega / k T)-1)
$$

given by the Bose-Einstein relation, where $\hbar$ is Planck's constant divided by $2 \pi$, and $\omega$ is the phonon angular frequency. A lower $T$ leads to less emission and therefore to less energy loss; hence, more electrons will reach the interface with high energy thus increasing $P_{J N}$.

However, in the low voltage regime even the energy of ballistic electrons $q \cdot V_{T O T}$ is not sufficient for injection over the barrier. Electrons may gain energies larger than $q \cdot V_{T O T}$ in absorbing energy from lattice phonons [3]. Under these conditions the distribution function at the interface should exhibit a so-called thermal tail above $q \cdot V_{T O T}$ with a slope equal to the lattice temperature, as suggested by numerical and analytical results $[3,6]$. Since in this case the number of phonon absorptions is proportional to $N_{\beta}$, at low 
applied $V_{T O T}$ one would expect $P_{J N}$ to decrease with decreasing $T$. This would lead to smaller $P_{J N}$ values at $77 \mathrm{~K}$ than at $300 \mathrm{~K}$, and $P_{J N}$ curves for different temperatures could cross each other (crossover).

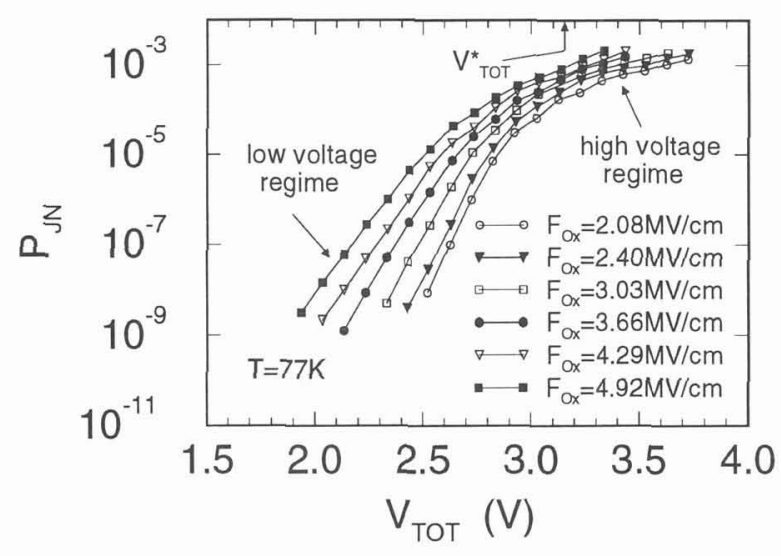

Figure 2: Measured injection probabilities for electrons at $77 \mathrm{~K}$ as a function of the total accelerating voltage.

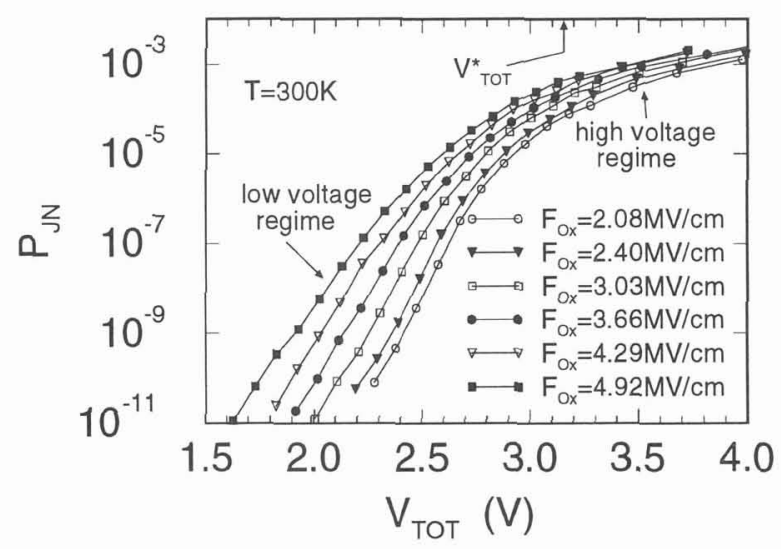

Figure 3: Measured injection probabilities for electrons at $300 \mathrm{~K}$ as a function of the total accelerating voltage.

Furthermore we notice, that injection from the thermal tail at two different $V_{T O T}$ can be described in a quasi thermal equilibrium approach [7] as

$$
P_{J N 1}=C\left(F_{O x}\right) \cdot \exp \left(-E_{t h} / k T\right) \quad P_{J N 2}=C\left(F_{O x}\right) \cdot \exp \left(-\left(E_{t h}-\Delta V_{T O T} \cdot q\right) / k T\right)
$$

where $C$ depends only on the oxide field, and $E_{t h}$ is the threshold energy for injection. Thus, the semilogarithmic slope of $P_{J N}$ against $V_{T O T}$ should return the lattice temperature

$$
\frac{\log \left(P_{J N 1}\right)-\log \left(P_{J N 2}\right)}{\Delta V_{T O T}}=\frac{-q}{\ln (10) \cdot k T} .
$$

However, if the above equation is used to interpret the low voltage regime measurements of Fig. 4, the extracted effective temperature $\left(T_{E F F}\right)$ ranges from about $500 \mathrm{~K}$ at $F_{O X}=2.08 \mathrm{MV} / \mathrm{cm}$ to about $700 \mathrm{~K}$ at 
$F_{O x}=4.92 \mathrm{MV} / \mathrm{cm}$ (see the shifted Maxwellian distributions in Fig. 4). The lattice temperature is definitely not reached. Furthermore, as seen in Fig. 4, no crossover occurs for the high field curves, whereas the crossover observed for the low field ones lies within the measurement tolerances. To explain the discrepancy between expected and measured $P_{J_{N}}$ we performed Monte Carlo simulations of the injection experiment with the established $\mathrm{MC}$ program BeBop, that was proved to provide quantitative agreement to $P_{J N}$ data at $300 \mathrm{~K}[8-10]$.

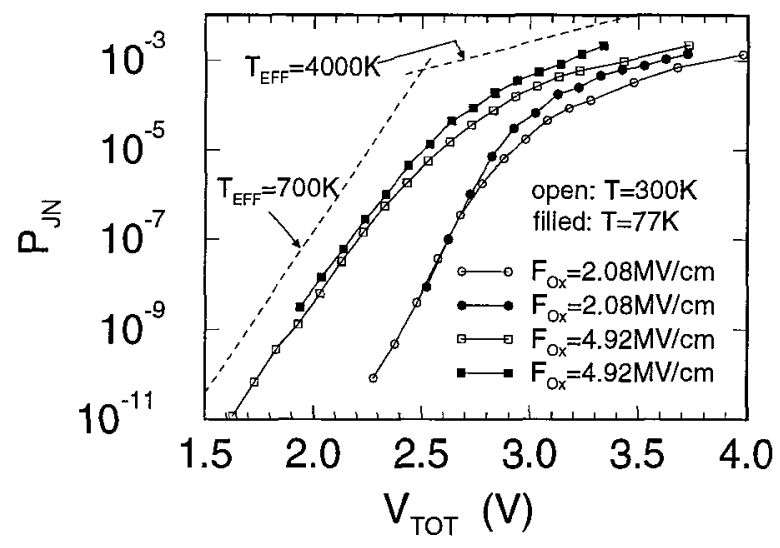

Figure 4: Direct comparison of measured $P_{J N}$ at the two different temperatures. Open symbols: $T=300 \mathrm{~K}$. Filled symbols: $T=77 \mathrm{~K}$. The dashed lines represent shifted Maxwellian distributions with $T_{E F F}=700 \mathrm{~K}$ and $T_{E F F}=4000 \mathrm{~K}$.

\section{THE SIMULATIONS}

In the simulations $P_{J N}$ can be calculated as seen in the inset of Fig. 5, with $f\left(E_{n}\right)$ the electron distribution at the interface (including the density of states) as a function of the normal electron energy, that is the energy computed from the normal component of the wavevector. Only electrons that move towards the interface contribute to this distribution. $P_{S i-G}$ is the transfer probability from the silicon to the gate, and $v_{g}$ the electron group velocity. The transfer probability (Fig. 5) is computed by solving the Schrödinger equation in the oxide [10] and, compared to the Fowler-Nordheim expression, provides a smooth transition between tunneling and thermionic emission by accounting for quantum reflections above the barrier. The Monte Carlo simulations included phonon scattering, impact ionization, impurity, carrier-carrier and plasmon scattering. Simulations performed with and without carrier-carrier and plasmon scattering provided the same results supporting the conclusion, that their influence is indeed negligible. For the sake of completeness, however, the following results will always include both of them.

Fig. 6 shows $f\left(E_{n}\right)$ for bias points in the high and low voltage regime. As expected [3], a thermal tail with an effective $T=77 \mathrm{~K}$ equal to the lattice temperature is observed at energies higher than $q \cdot V_{\text {ToTin, }}$, which is mostly due to phonon absorption [3]. Furthermore a peak due to ballistic electrons can be seen at $\mathrm{E}_{\mathrm{n}}=\mathrm{q} \cdot \mathrm{V}_{\mathrm{TOT} 1 / 2}$.

Compared to $f$ and $P_{S i-G}, v_{g}$ is a weak function of energy. Thus; the distribution of the injected electrons is essentially given by $f \cdot P_{S i-G}$ (also shown in Fig. 6). The two different injection regimes can be explained by analyzing the maxima of the $f \cdot P_{S i-G}$ curves, that indicate the energy range in which injection occurs. In the high voltage regime $\left(V_{T O T 2}\right)$ electrons are injected only from the high effective temperature part of the distribution around $\Phi_{B}$, leading to the extraction of a high $T_{E F F}$ from $P_{J N}$ versus $V_{T O T}$ data. In the low voltage regime $\left(V_{T O T 1}\right)$ the average energy of the injected electrons is approximately $q \cdot V_{T O T 1}$, that is much smaller than $\Phi_{B}$, due to the rapidly decreasing number of electrons in the thermal tail. As a consequence, injection is not only due to electrons in the tail, that gained additional energy by phonon absorption, but 
also by the electrons in the high effective temperature part of $f\left(E_{n}\right)$, that tunnel to the floating gate from energy levels well below $\Phi_{B}$. Although very few, these electrons are still much more than those populating the tail. Thus, they give a relevant contribution to injection. This explains why $T_{E F F}$ does not reach the lattice temperature, and why the crossover effect is not much pronounced even at the lowest $V_{T O T}$ in the measurements (Fig. 4). The contribution to the gate current of electrons with energy below $q \cdot V_{T O T}$ becomes even larger for increasing oxide fields (or thinner oxides) because of the more shallow slope of $P_{S i-G}\left(E_{n}\right)$, and this explains why the $P_{J N}$ slope decreases with increasing field in the low voltage regime.

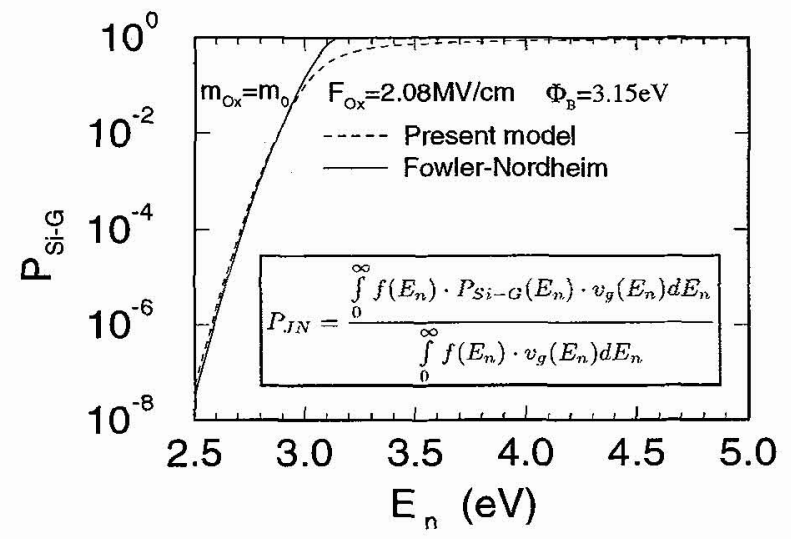

Figure 5: Transfer probabilities computed according to the Fowler-Nordheim tunneling formula (solid line), and to the model [10] used in the MC simulations (dashed line), for one of the oxide fields of the experiments. Inset: equation for $P_{J N}$ used to interpret the simulation results.

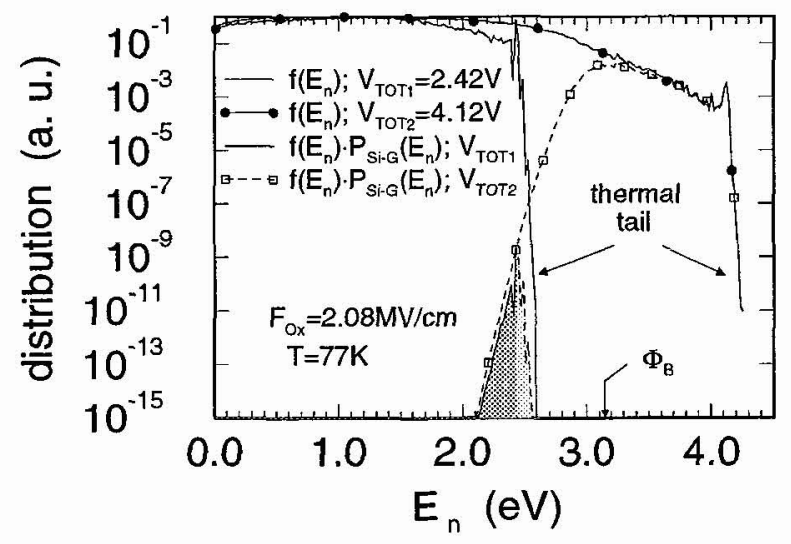

Figure 6: Solid lines: simulated $f\left(E_{n}\right)$ in the low $\left(V_{T O T 1}\right)$ and the high $\left(V_{T O T 2}\right)$ voltage regime. Dashed lines: simulated distribution of the injected electrons $f \cdot P_{S i-G}$. The bright filling under the $f \cdot P_{S i-G}$ curve for $V_{\text {TorI }}$ shows the contribution to $P_{J N}$ of the thermal tail, the dark filling the contribution of the high effective temperature part of the distribution function.

We would like to point out that the presence of additional scattering mechanisms not considered in this work may smear out the sharp transition in the distribution functions of Fig. 6, but will not upset the 
qualitative picture outlined here, so long as $f\left(E_{n}\right)$ decreases above $q \cdot V_{T o r}$ more rapidly than the increase in $P_{S i-G}$. Finally we notice that the lucky carrier model and the thermal equilibrium approach based on Maxwellian distributions with a unique effective temperature cannot reproduce the sharp transition in the distributions of Fig. 6, and hence will fail to predict the two different regimes evident by the data in Fig. 2 and Fig. 3.

\section{Acknowledgements}

The authors would like to thank R. Bez (SGS Thomson, Milano) for the fabrication of the devices used in the experiments. One of the authors (B. F.) gratefully acknowledges the financial support by the European SUSTAIN network under contract no. ERBCHRXT920060.

\section{References}

[1] Ning T. H., Osburn C. M., and Yu H. N., J. Appl. Phys. 48 (1976) 286-293.

[2] Esseni D., et al., IEEE Electron Device Lett. 16 (1995) 506-508.

[3] Abramo A., Fiegna C., and Venturi F., "Hot carrier effects in short MOSFETs at low applied voltages," IEDM, Washington, DC 10-13 December 1995, pp. 301-304.

[4] Selmi L., et al., "A test chip and an accurate measurement system to characterize hot hole injection in the gate oxide of p-MOSFETs," Proceedings ICMTS, San Diego, CA 22-25 March 1994, pp. 68-71.

[5] Yu Z., Chen D., So L., and Dutton R. W., "PISCES-2ET," Stanford University Integrated Circuits Laboratory Technical Report, 1994.

[6] Mahan G. D., J. Appl. Phys. 58 (1985) 2242-2251.

[7] Tam S., et al., IEEE Electron Device Lett. 4 (1983) 249-251.

[8] Abramo A., et al., J. Appl. Phys. 76 (1994) 5786-5794.

[9] Abramo A., et al., IEEE Trans. Computer Aided Design 12 (1993) p. 1327-1336.

[10] Ghetti A., et al., "A combined transport-injection model for hot-electron and hot-hole injection in the gate oxide of MOS structures," IEDM, San Francisco, CA 11-14 December 1994, pp. 363-366. 